Supporting information

\title{
Nano-projectile secondary ion mass spectrometry for analysis of extracellular vesicles
}

D. S. Verkhoturov', B.P. Crulhas' ${ }^{2}$, M. J. Ellerit, Y. D. Han', S.V. Verkhoturov', Y.

\author{
Bisrat $^{4}$, A. Revzin ${ }^{2}$ and E. A. Schweikert ${ }^{1}$
}

${ }^{1}$ Department of Chemistry, Texas A\&M University, College Station, Texas 77843, USA

${ }^{2}$ Department of Physiology and Biomedical Engineering, Mayo Clinic, 200 1st Street SW St-11-14, Rochester, MN 55905, USA

${ }^{3}$ Department of Chemistry and Biochemistry, California State University Northridge, Northridge, CA

$$
\text { 91330, USA; }
$$

${ }^{4}$ Materials Characterization Facility, Texas A\&M University, College Station, Texas 77843, USA;

†Correspondence to: michael.eller@csun.edu

Figure S1. Fluorescence microscopy image of EV surface.

Figure S2. UV-VIS spectrum of each antibody-tag conjugate

Figure S3. UV-VIS standard curves for each tag ................................................................................. 3

Figure S4. The nanoparticle tracking analysis of isolated EVs .......................................................... S4

Figure S5. Selected regions of the mass spectrum of EVs tagged with BHHTEGST and erythrosine

Figure S6. Two-dimensional correlation heat map from a multi-layer EV deposit tagged with anti-CD81-

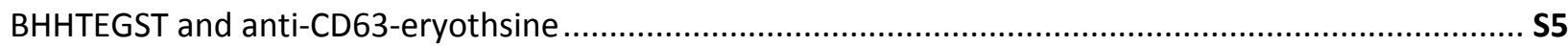

Figure S7. Two-dimensional correlation heat map from a multi-layer EV deposit non-tagged ............... S5

Figure S8. SEM micrograph of dispersed EVs at 3500X magnification and 18000X magnification............ S6

Figure S9. Selected region of the mass spectrum of dispersed tagged EVs ............................................ 6

Figure S10. Selected region of the mass spectrum of dispersed tagged EVs .......................................S7

Figure S11. Selected region of the mass spectrum of dispersed tagged EVs ........................................S7

Figure S12. Two-dimensional correlation heat map for anti-CD81-BHHTEGST tagged EVs ................... S8 


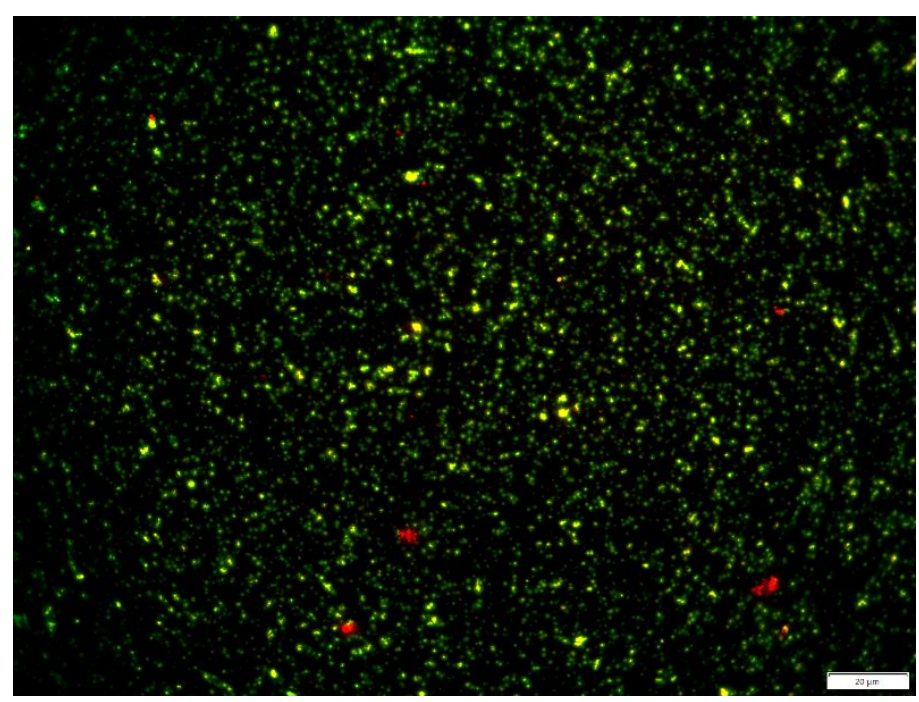

Figure S1. Fluorescence microscopy image $532 \mathrm{~nm}$ excitation $/ 548 \mathrm{~nm}$ emission of erythrosineanti-CD63 labeled DIO EVs captured via electrostatic interactions on a poly-L-lysine-coated ITO glass slide.

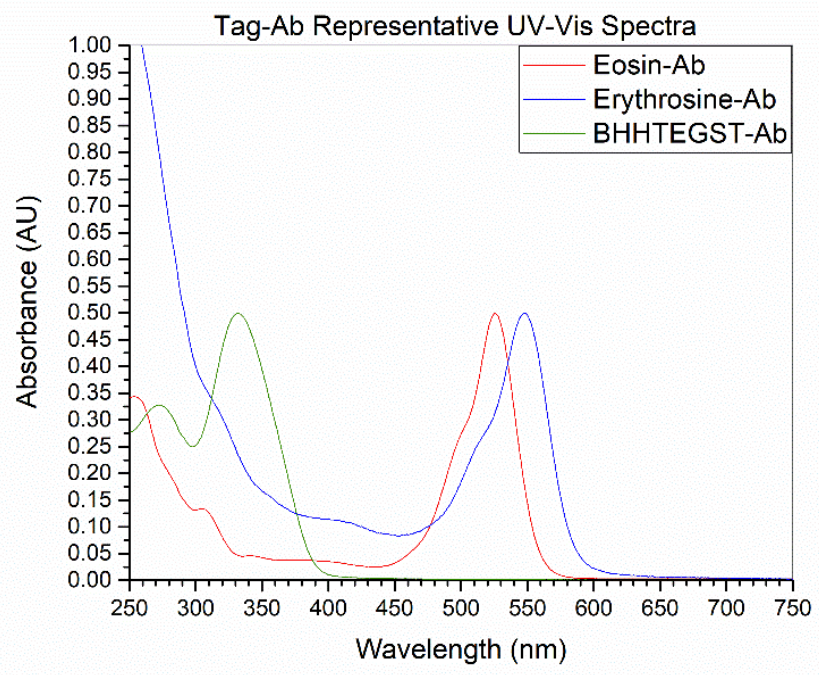

Figure S2. Upon conjugation of tags with Ab, products were characterized via UV-Vis. BHHTEGEST, erythrosine and eosin have characteristic absorption maxima at $330 \mathrm{~nm}, 558 \mathrm{~nm}$, and $540 \mathrm{~nm}$ respectively. 

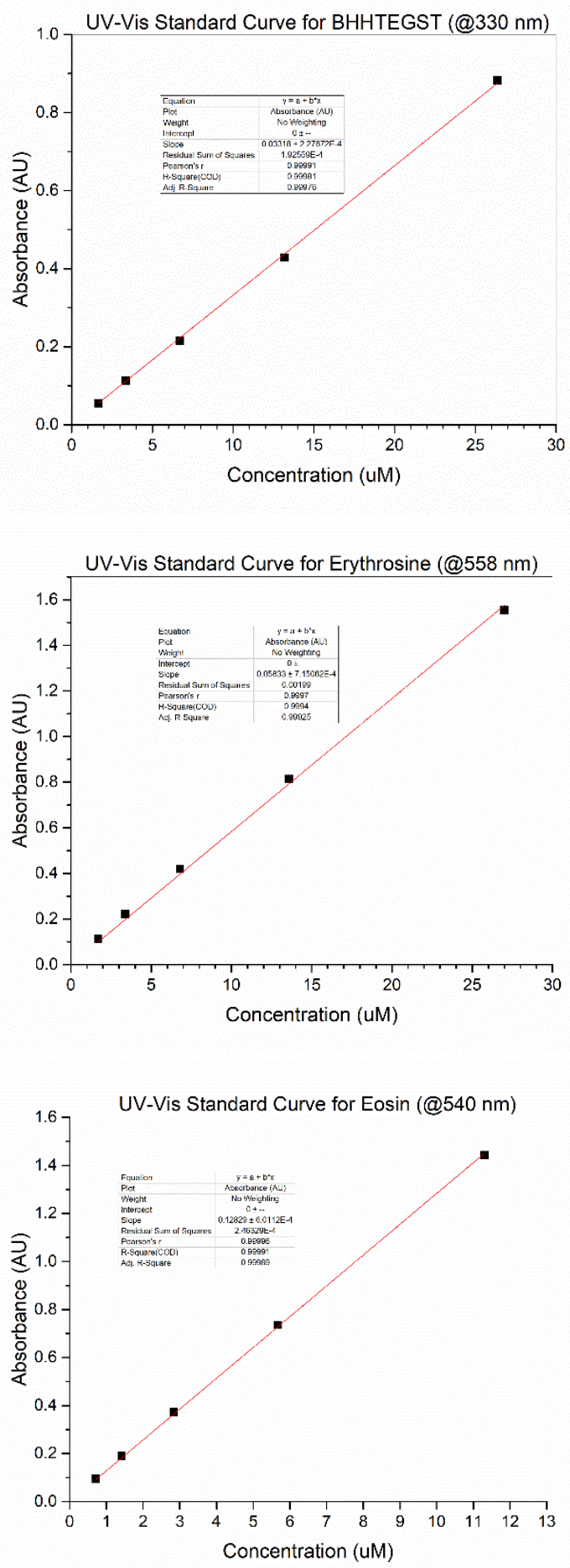

Figure S3. Standard curves were calculated for each tag at the characteristic wavelength.

Using the standard curves, the tag concentration of the product was calculated. Then to calculate the molar ratio of tag to $A b$, the $A b$ concentration must be calculated using the peak at $280 \mathrm{~nm}$. However, there is interference from the tag at that wavelength. The interference was calculated using UV-Vis spectra of the flowthrough during the cleaning step because the flowthrough contains only the tag in 
the same buffer environment as the product without the presence of the Ab. For BHHTEGST the interference was $40 \%$. The conjugation protocol was optimized based on this characterization method.

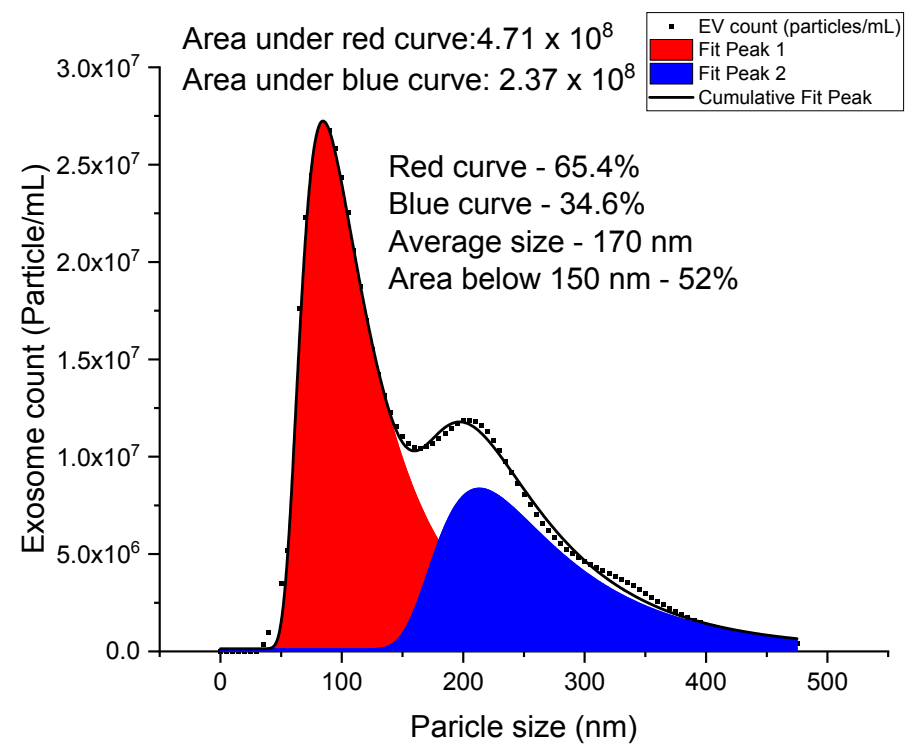

Figure S4. The nanoparticle tracking analysis (NTA) was performed using a Nanosight NS300 (Malvern Panalytical, Malvern, UK) in the Microscopy and Cell Analysis Core at Mayo Clinic Rochester. Particle dimensions and size distribution were exported into and analyzed using OriginPro. The two peaks in the distribution were fit the pulse peak shape.

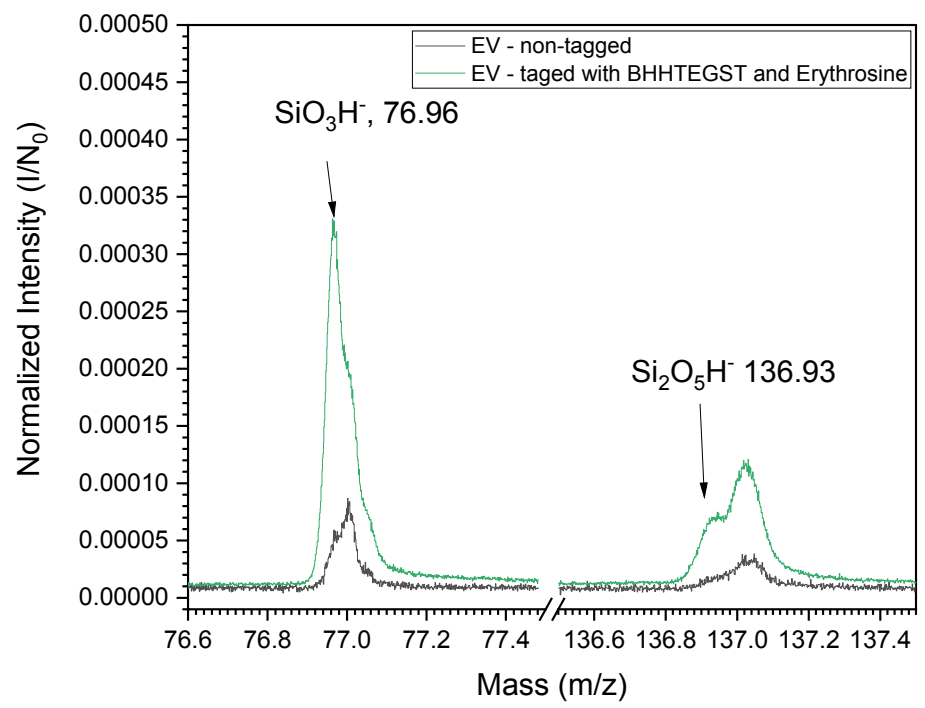

Figure S5. Selected regions of the mass spectrum of EVs tagged with BHHTEGST and erythrosine (green trace), compared to EVs without tag (black trace). 


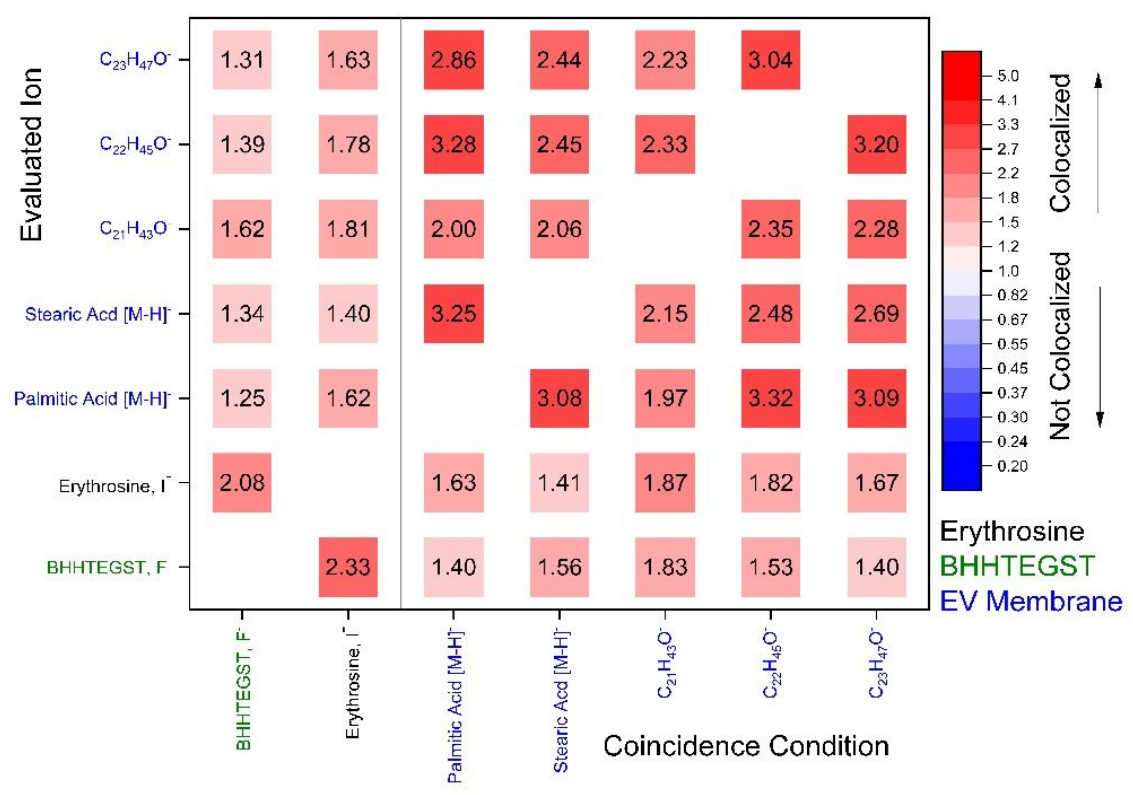

Figure S6. Two-dimensional correlation heat map from a multi-layer EV deposit tagged with anti-CD81-BHHTEGST and anti-CD63-eryothsine. The color scale is the correlation coefficient, with red corresponding to positive correlation and blue to negative correlation. The evaluated ions are listed on the $y$-axis, and the selected ion is listed on the $x$-axis.

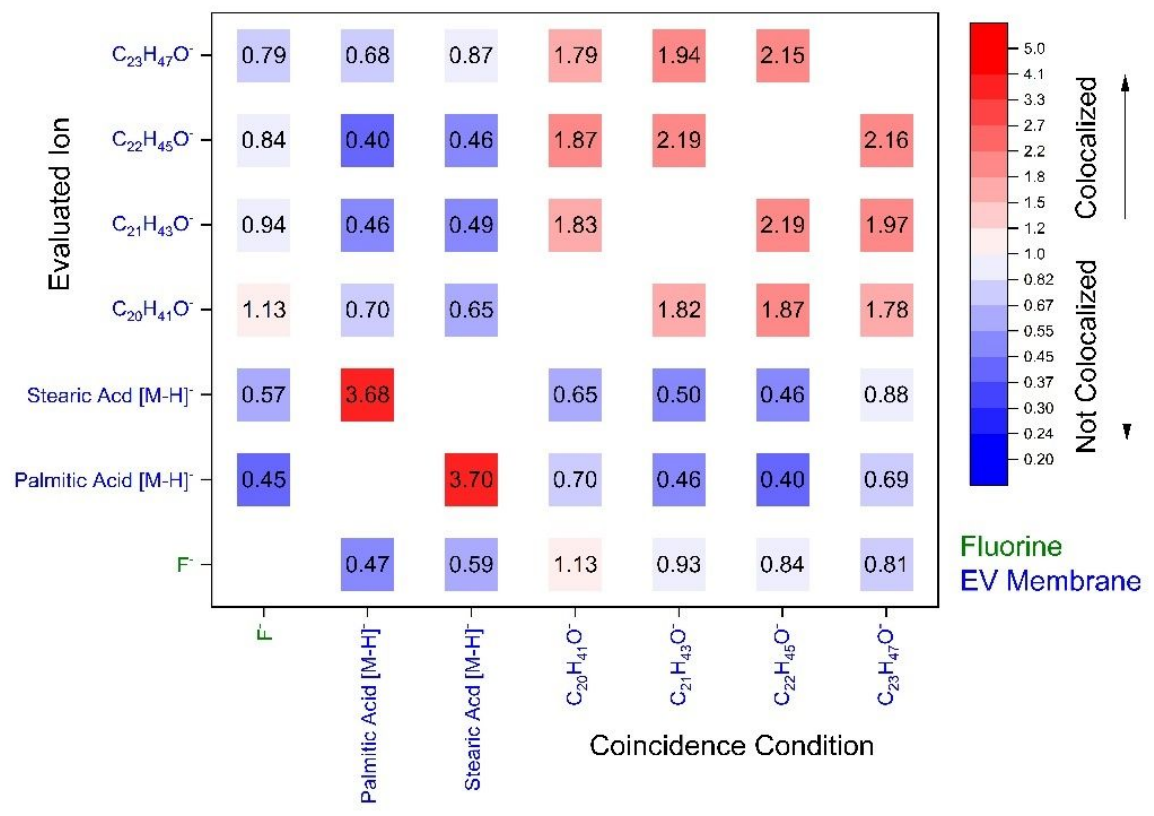

Figure S7. Two-dimensional correlation heat map from a multi-layer EV deposit non-tagged. The color scale is the correlation coefficient, with red corresponding to positive correlation and blue to negative correlation. The evaluated ions are listed on the $y$-axis, and the selected ion is listed on the $x$-axis. Note that the signal due to fluorine is due to surface contamination. 

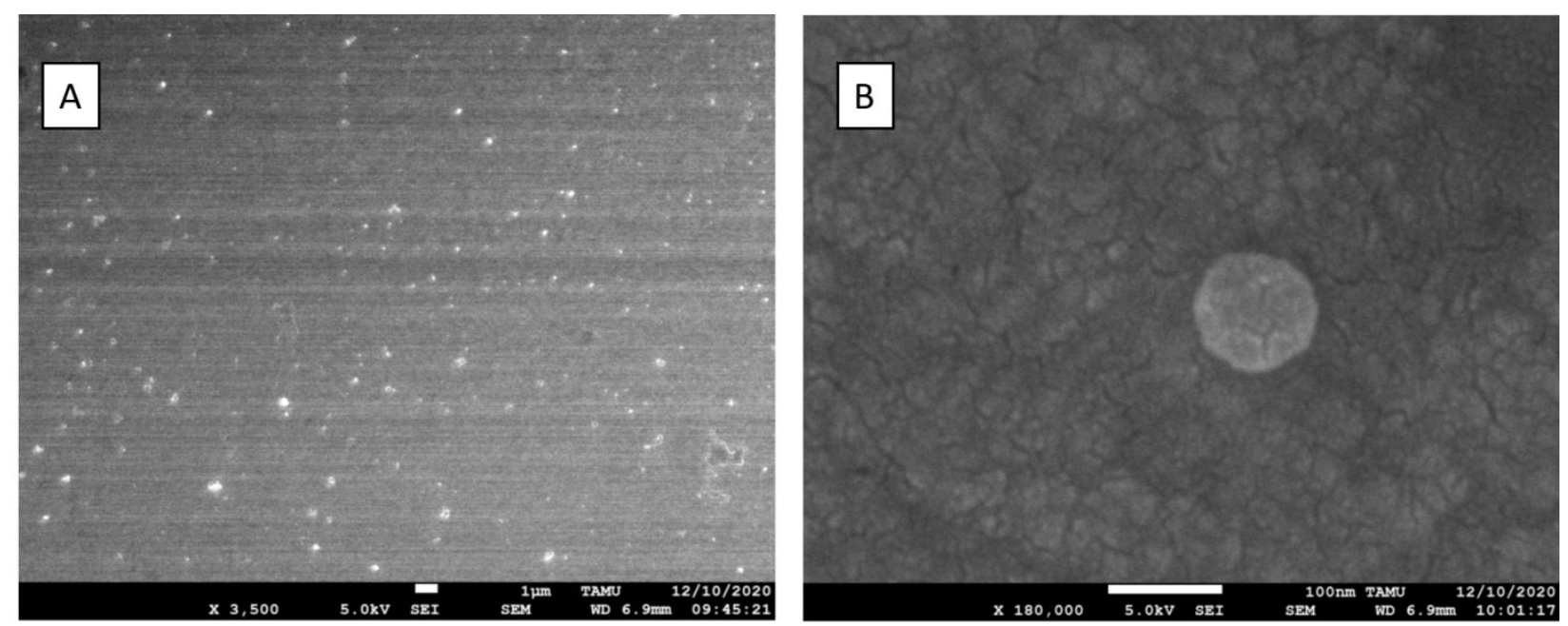

Figure S8. A) SEM micrograph of isolated EVs at 3500X magnification. B) SEM micrograph of isolated EV at 180,000X magnification. The isolated EVs were analyzed with SEM. The SEM results are presented in figure 7A and 7B. As can be seen in the SEM magnification micrograph (Figure 7A) EVs were successfully dispersed on the surface. A high magnification image of one of the EVs (Figure 7B) shows an example of an intact isolated EV.

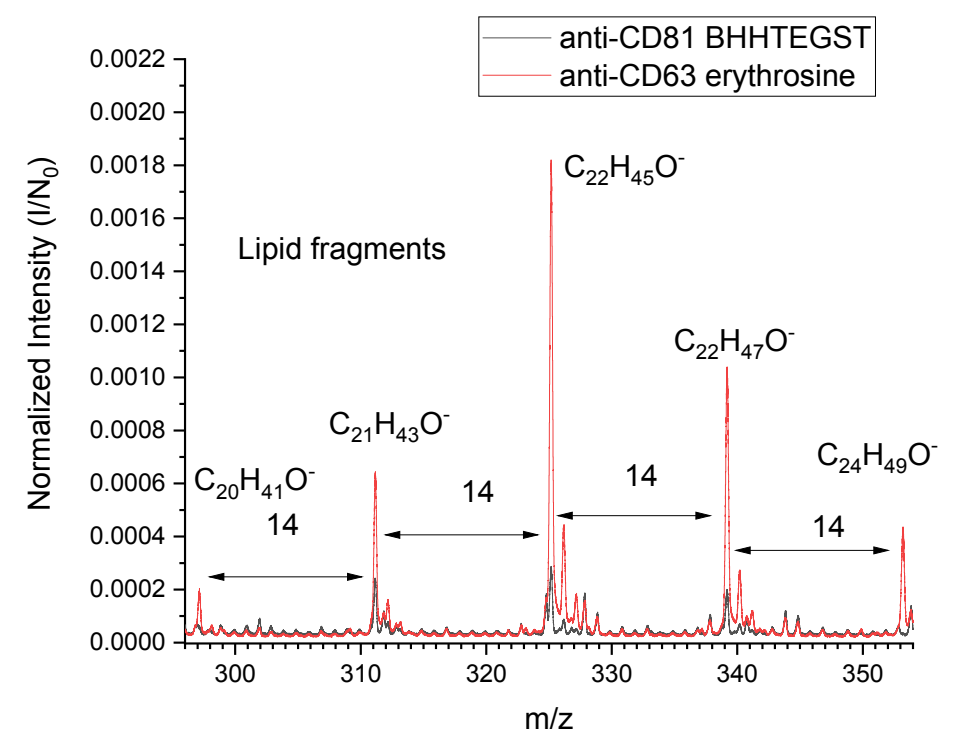

Figure 59. Selected region of the mass spectrum of dispersed EVs tagged with anti-CD63 erythrosine (red trace) and EVs tagged with anti-CD81 BHHTEGST (black trace). 


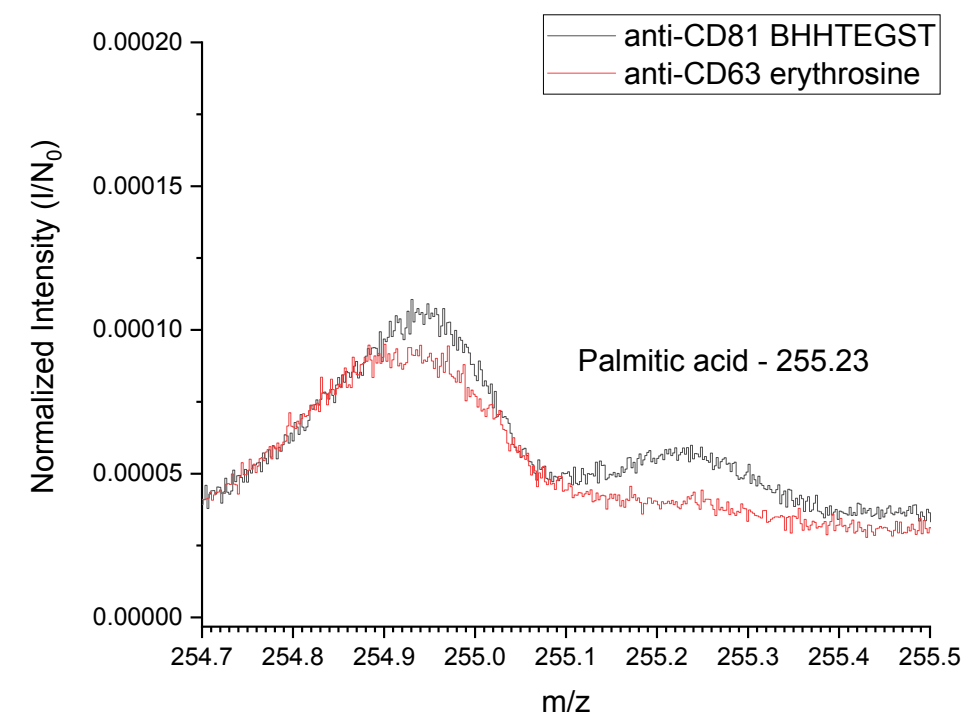

Figure S10. Selected region of the mass spectrum of dispersed EVs tagged with anti-CD63 erythrosine (red trace) and EVs tagged with anti-CD81 BHHTEGST (black trace).

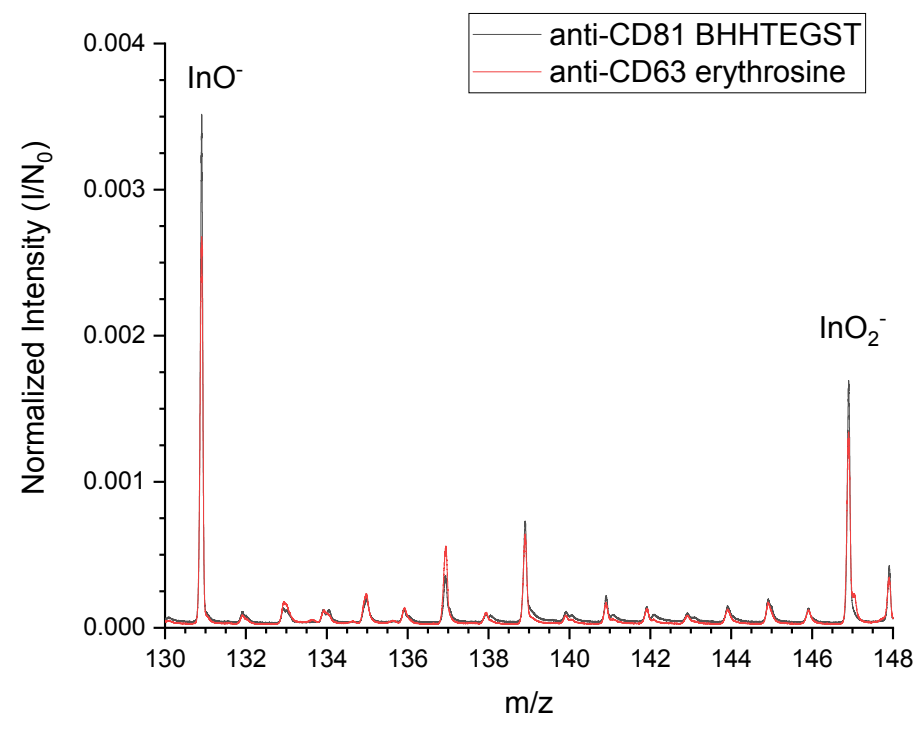

Figure S11. Selected region of the mass spectrum of dispersed EVs tagged with anti-CD63 erythrosine (red trace) and EVs tagged with anti-CD81 BHHTEGST (black trace). 


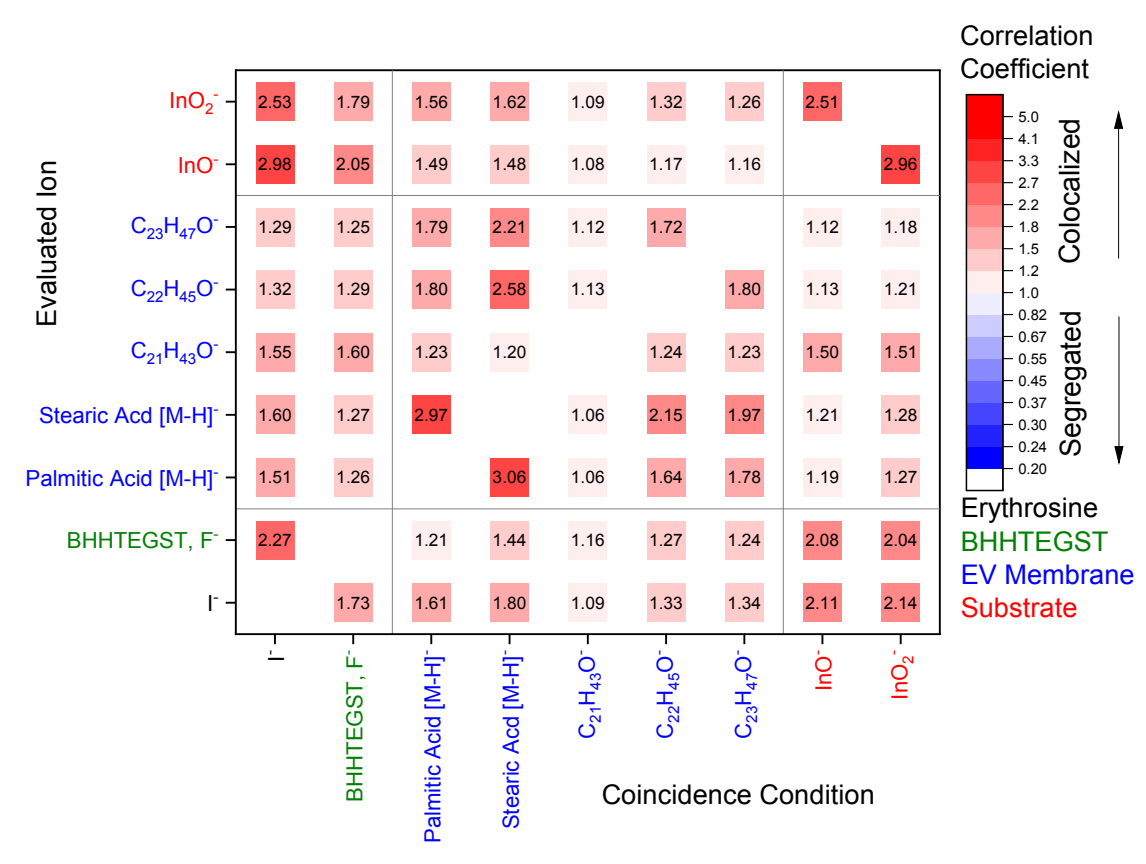

Figure S12. Two-dimensional correlation heat map from dispersed anti-CD81-BHHTEGST tagged EVs. The color scale is the correlation coefficient, with red corresponding to positive correlation and blue to negative correlation. The evaluated ions are listed on the $y$-axis, and the selected ion is listed on the $x$-axis. 